\title{
Increasing Knowledge on Post-Acute Rehabilitation in COVID-19
}

\author{
Massimiliano Polastri \\ Department of Continuity of Care and Disability, Physical Medicine and Rehabilitation, St Orsola University Hospital, \\ Bologna, Italy
}

\section{Dear Editor,}

I read with interest the study by Zampogna et al. [1], where the authors described their clinical experience with a cohort of patients recovering from COVID-19 enrolled in a rehabilitation programme. As highlighted by the authors, at the beginning of the pandemic very little information was available regarding the rehabilitative treatment in such an unprecedented situation apart from practical recommendations released by scientific/professional societies [2]. Thanks to those recommendations, healthcare professionals involved in the rehabilitation treatment of patients with COVID-19 have found a scientific basis for approaching patients in both acute and subacute care settings. Since the first wave of the pandemic occurred in early 2020, only a small number of studies discussing physiotherapy practice have been published when compared with thousands of articles in the medical field. From a certain point of view, the study by Zampogna et al. [1] can be considered one of the firsts including a considerable number of patients via a retrospective observational design. Another previously published study by Jiandani et al. [3] investigated the effects of rehabilitation in COVID-19 in a broader population using the same study design. If we would reflect on the paucity of studies discussing rehabilitation in COVID-19, we have to consider the following arguments: (1) it seems that observational designs are encountering difficulties being published probably because they are typically considered a type of research bearing the bias of being uncontrolled in nature. (2) We all as clinicians, researchers, authors, reviewers, and editors are aware of the increasing interest mounting on the rehabilitation field, but we observe that this topic - at the time of writing - is underestimated. (3) Is rehabilitation considered a secondary therapeutic line of treatment not deserving the same attention which is given to other disciplines?

All these hypotheses should be considered when reading the study by Zampogna et al. [1] who have produced research containing valuable insights. In fact, from their article, it can be gathered - among the various information available - that patients can afford a rehabilitation treatment (whose intensity varied according to their clinical conditions) when having a $\mathrm{PaO}_{2} / \mathrm{FiO}_{2}$ ratio $\geq 310 \mathrm{~mm}$ $\mathrm{Hg}$, after a long hospitalization $\geq 33.5$ days, and being able to walk $\geq 250 \mathrm{~m}$ at the 6 -min walking test after having attended a rehabilitation programme [1]. Let me say that these are not secondary aspects. All these pieces of information are available from a retrospective observational study design.

Another message that can be taken from the experience of Zampogna et al. [1] concerns the obvious reflection on the impossibility to conduct studies with control 
populations because, in that case, patients could be exposed to the risk of not receiving rehabilitation.

The study by Zampogna et al. [1] provides helpful insights to be transferred to the real world. It could contribute to inspiring authors, reviewers, and editors in producing, revising, and publishing more pieces of research considering that observational studies of rehabilitation in COVID-19 are probably the unique viable option if we want to expand our knowledge in this specific matter.

\section{Conflict of Interest Statement}

The author has no conflicts of interest to declare.

\section{Funding Sources}

No funding was received.

\section{References}

1 Zampogna E, Paneroni M, Belli S, Aliani M, Gandolfo A, Visca D, et al. Pulmonary rehabilitation in patients recovering from COVID-19. Respiration. 2021;100(5):41622.
2 Polastri M, Brini S, Ghetti A, Lama A. Recommendations form scientific/professional societies: an essential support for physiotherapy in patients with COVID-19. Int J Ther Rehabil. 2020;27(4):1-3.
3 Jiandani MP, Salagre SB, Kazi S, Iyer S, Patil P, Khot WY, et al. Preliminary observations and experiences of physiotherapy practice in acute care setup of COVID 19: a retrospective observational study. J Assoc Physicians India. 2020;68(10):18-24. 